\title{
Adaptive Second-Order Sliding Mode Control Design for a Class of Nonlinear Systems with Unknown Input
}

\author{
You Zheng, ${ }^{1}$ Jianxing Liu, ${ }^{2}$ Xinyi Liu, ${ }^{3}$ Dandan Fang, ${ }^{4}$ and L. Wu ${ }^{1}$ \\ ${ }^{1}$ Ningbo University of Technology, Ningbo 315000, China \\ ${ }^{2}$ School of Astronautics, Harbin Institute of Technology, Harbin 150000, China \\ ${ }^{3}$ Laboratoire OPERA, Universite de Technologie de Belfort-Montbeliard, 90010 Belfort, France \\ ${ }^{4}$ Ningbo Academy of Smart City Development, Ningbo 315000, China \\ Correspondence should be addressed to Jianxing Liu; jx.liu@hit.edu.cn
}

Received 7 March 2015; Accepted 20 April 2015

Academic Editor: Xinggang Yan

Copyright (C) 2015 You Zheng et al. This is an open access article distributed under the Creative Commons Attribution License, which permits unrestricted use, distribution, and reproduction in any medium, provided the original work is properly cited.

\begin{abstract}
An adaptive second-order sliding mode controller is proposed for a class of nonlinear systems with unknown input. The proposed controller continuously drives the sliding variable and its time derivative to zero in the presence of disturbances with unknown boundaries. A Lyapunov approach is used to show finite time stability for the system in the presence of a class of uncertainty. An illustrative simulation example is presented to demonstrate the performance and robustness of the proposed controller.
\end{abstract}

\section{Introduction}

Sliding mode control (SMC) has gained much attention due to its attractive characteristics of finite time convergence and robustness against uncertainties [1-3]. Sliding mode control has been thoroughly studied, from both practical and theoretical point of view, for example, successfully applied for control of time delay systems $[4,5]$, control and observation of fuel cell power systems [6-11], control and observation of power converters [12-16], and adaptive control of flexible spacecraft $[17,18]$. Robustness of a control system is essential based on the reason that various uncertainties exist in practical systems. These nonlinear systems are with structured and unstructured uncertainties and external disturbances such as load variation. However, the price of using sliding mode control to achieve robustness to these disturbances is control chattering problem [19-22]; chattering is undesired because it may cause high frequency dynamics and even instability. Therefore, in order to achieve the optimal operation performance of such uncertain systems, suitable model and controller development efforts are needed [23-26].

There are several ways to avoid chattering problem when using sliding mode control. The conventional SMC uses a control law with large control gains yielding the undesired chattering while the control system is in the sliding mode. To eliminate the chattering, the discontinuous control function is replaced by "saturation" or continuous "sigmoid" functions in $[27,28]$. However, such approach constrains the sliding system's trajectories not to the sliding surface but to its vicinity losing the robustness to the disturbances. Higher order sliding mode control techniques are used in [29-32] which allow driving the sliding variable and its consecutive derivatives to zero in the presence of the disturbances. However, the main challenge of high order sliding mode controllers is the use of high order time derivatives of sliding variable. It is interesting to note that the popular supertwisting algorithm [1] only requires the measurement of the sliding mode variable without its time derivative. Recently, adaptive sliding mode controller has been proposed to tune the controller gains with respect to disturbances [33, 34]. Intelligent controllers such as fuzzy neural network [35, $36]$ and adaptive fuzzy sliding mode controller [37, 38] were proposed to reduce the chattering. However, all the aforementioned literatures address the case based on the assumption that the boundary of the disturbances is known. This boundary cannot be easily obtained in practical cases. 
The overestimating of the disturbance boundary yields to larger than necessary control gains, while designing the super-twisting control law [32].

In this paper, an adaptive controller gain super-twisting algorithm (ASTW) is proposed for nonlinear system with unknown input. Based on the Lyapunov theory, the proposed control law continuously drives the sliding variable and its time derivative to zero in the presence of bounded unknown input but without knowing the boundary. The stability and the robustness of the control system are proven, and the tracking performance is ensured.

\section{Problem Formulation}

The super-twisting control law (STW) is effective to remove the chattering when the relative degree equals one. It generates the continuous control function that drives the sliding variable and its time derivative to zero in finite time in the presence of bounded unknown disturbance. The main disadvantage of STW algorithm is that it requires the bounded value of $\dot{\sigma}$ [39] ( $\sigma$ is the sliding variable). Unfortunately, the knowledge of $\dot{\sigma}$ is often unavailable. The overestimating of the $\dot{\sigma}$ will yield to larger than necessary control gains while designing the STW control law. In this note, an adaptive-gain approach will be adopted when designing STW control law, which does not require the bounded value of $\dot{\sigma}$. The idea of adaptive-gain approach is to increase the control gain $\alpha, \beta$ dynamically until the STW controller converges. Once the $\sigma>0$, then gains will start reducing; this gains reduction will be reversed as soon as the sliding variable $\sigma$ and its time derivative $\dot{\sigma}$ start deviating from the equilibrium point $\sigma=$ $\dot{\sigma}=0$.

Consider a class of $n$ th-order uncertain nonlinear system, which is represented in a state-space form as

$$
\dot{x}=f(x)+b(x) u+d,
$$

where $x=\left[x_{1}, x_{2}, \ldots, x_{n}\right]^{T}=\left[x_{1}, \dot{x}_{1}, \ldots, x_{1}{ }^{n-1}\right]^{T} \in \mathbb{R}^{n}$ is the state vector, $u \in \mathbb{R}$ is the control function, $d \in \mathbb{R}^{n}$ is the external disturbance, and $f(x) \in \mathbb{R}^{n}$ and $b(x) \in \mathbb{R}^{n}$ are the smooth vector fields.

Let the desired state vector be $x_{d}$; the tracking error and the sliding-surface function are defined as

$$
\begin{aligned}
& e(x, t)=x-x_{d} \\
& \sigma(x, t)=c^{T} e
\end{aligned}
$$

where $c=\left[c_{1}, c_{2}, \ldots, c_{n}\right]^{T}$. The sliding mode will be obtained in finite time if an appropriate control law is applied. In the sliding mode, the error dynamics will be

$$
c_{n} e_{1}^{(n-1)}+c_{n-1} e_{1}^{(n-2)}+\cdots+c_{1} e_{1}=0
$$

The constants $c_{1}, c_{2}, \ldots, c_{n}$ are chosen to be positive such that the eigenvalue polynomial $\varphi(\lambda)=c_{n} \lambda^{n-1}+c_{n-1} \lambda^{n-2}+\cdots+c_{1}$ is Hurwitz. The choice of $c$ decides the convergence rate of the tracking error.
Assumption 1. The relative degree of system (1) with the sliding variable $\sigma$ with respect to $u$ equals one; in other words, the control function $u$ has to appear explicitly in the first total derivative $\dot{\sigma}$.

Under Assumption 1, the dynamics of $\sigma$ can be computed as follows:

$$
\dot{\sigma}=g(x) u+\rho(x, t)
$$

where $\rho(x, t)=c^{T} f(x)+c^{T} d-c^{T} \dot{x}_{d}$ and $g(x)=c^{T} b(x) \neq 0$.

Assumption 2. The first-order time derivative of the uncertain function $\rho(x, t) \in \mathbb{R}$ is bounded

$$
\dot{\rho}(x, t) \leq \delta,
$$

for some unknown constants $\delta>0$.

Assumption 3. The uncertain function $g(x, t) \in \mathbb{R}$ is presented as

$$
g(x, t)=g_{0}(x, t)+\Delta g(x, t),
$$

where $g_{0}(x, t)>0$ is the nominal parts of $g(x, t)$ and $\Delta g(x, t)$ is the parameter variation such that

$$
\left|\frac{\Delta g(x, t)}{g_{0}(x, t)}\right| \leq \eta<1 .
$$

With Assumptions 2 and 3, (4) can be written as follows:

$$
\dot{\sigma}=\rho(x, t)+g_{1}(x, t) \omega,
$$

where $1-\eta \leq g_{1}(x, t)=1+\Delta g(x, t) / g_{0}(x, t) \leq 1+\eta$ and $\omega=g_{0}(x, t) u$. The solution of $(8)$ is understood in the sense of Filippov [40].

\section{Adaptive-Gain STW Controller Design}

The control objective is to drive the sliding variable $\sigma$ and its derivative $\dot{\sigma}$ to zero in finite time without the control gain overestimation based on Assumptions 2 and 3 satisfied. The classical SMC can handle with the task to keep an output variable $\sigma$ at zero when the relative degree of $\sigma$ is one. However, the high frequency control switching leads to the chattering effect which is exhibited by high frequency vibration of the controlled plant and can be dangerous in some applications. The second-order sliding mode (SOSM) controllers including the continuous STW control algorithm are able to remove the chattering effect while preserving the main sliding mode features and improving its accuracy in the presence of unknown disturbance. However, it requires the knowledge of bounded disturbance. Unfortunately, the assumption of the disturbance is often unavailable in practice which leads to controller gain overestimation.

In this note, an adaptive-gain approach is used to solve this problem with STW algorithm. STW controller generates the continuous control function to remove the chattering effect while adaptive-gain approach allows controller gain nonoverestimation. 
The STW algorithm can be described by differential inclusion as follows [1,32]:

$$
\begin{aligned}
& \omega=-\lambda|\sigma|^{1 / 2} \operatorname{sign}(\sigma)+v \\
& \dot{v}=-\alpha \operatorname{sign}(\sigma),
\end{aligned}
$$

where the time varying controller gains

$$
\begin{aligned}
& \lambda=\lambda(\sigma, t), \\
& \alpha=\alpha(\sigma, t)
\end{aligned}
$$

are to be defined later.

Substituting (9) into (8), it follows that

$$
\begin{aligned}
& \dot{\sigma}=-\lambda g_{1}(x, t)|\sigma|^{1 / 2} \operatorname{sign}(\sigma)+\varphi, \\
& \dot{\varphi}=-\alpha g_{1}(x, t) \operatorname{sign}(\sigma)+\phi(x, t),
\end{aligned}
$$

where $\varphi=g_{1}(x, t) v+\rho(x, t)$ and $\phi(x, t)=\dot{g}_{1}(x, t) v+\dot{\rho}(x, t)$.

The term $\dot{g}_{1}(x, t) v$ is assumed to be bounded with some unknown constant $\delta_{1}$; that is,

$$
|\phi(x, t)| \leq \delta_{1}+\delta=\delta_{2},
$$

for some unknown constant $\delta_{2}>0$.

In this paper, the gains $\lambda(\sigma, t)$ and $\alpha(\sigma, t)$ are formulated as

$$
\begin{aligned}
& \lambda(\sigma, t)=\lambda_{0} \sqrt{l(t)}, \\
& \alpha(\sigma, t)=\alpha_{0} l(t),
\end{aligned}
$$

with $\lambda_{0}, \alpha_{0}$ arbitrary positive constants and a positive time varying scalar $l(t)$. The dynamic law of the varying function $l(t)$ is given by

$$
i(t) \begin{cases}k, & \text { if }|\sigma| \neq 0 \\ 0 . & \text { otherwise }\end{cases}
$$

where $k>0$ is a positive constant.

Now, the control objective is reduced to driving $\sigma$ and its derivative $\dot{\sigma}$ by (13) and (14) to zero in finite time with the condition of bounded perturbations in (12). Thus, the design of adaptive STW controller is formulated in the following theorem.

Theorem 4. Consider system (11); suppose that (12) holds. Then, for any initial conditions $x(0), \sigma(0)$, the adaptive-gain STW control laws (13), (14) drive the sliding variable $\sigma$ and its derivative $\dot{\sigma}$ to zero in finite time.

Proof. A new state vector is introduced in order to present system (11) in a more convenient form for Lyapunov analysis:

$$
\zeta=\left[\begin{array}{l}
\zeta_{1} \\
\zeta_{2}
\end{array}\right]=\left[\begin{array}{c}
l^{1 / 2}(t)|\sigma|^{1 / 2} \operatorname{sign}(\sigma) \\
\varphi
\end{array}\right] ;
$$

thus, system (11) can be rewritten as

$$
\dot{\zeta}=\frac{l}{2\left|\zeta_{1}\right|} \underbrace{\left[\begin{array}{cc}
-\bar{\lambda}_{0} & 1 \\
-2 \bar{\alpha}_{0} & 0
\end{array}\right]}_{A} \zeta+\left[\begin{array}{c}
0 \\
\phi(x, t)
\end{array}\right]+\left[\begin{array}{c}
\frac{i(t)}{2 l(t)} \zeta_{1} \\
0
\end{array}\right],
$$

where $\bar{\lambda}_{0}=\lambda_{0} g_{1}(x, t)$ and $\bar{\alpha}_{0}=\alpha_{0} g_{1}(x, t)$. Given that $g_{1}(x, t)>0$, it is easy to verify that $A$ is a Hurwitz matrix.

Then, the following Lyapunov function candidate is introduced for system (16):

$$
\begin{aligned}
& V=\zeta^{T} P \zeta \\
& P=\frac{1}{2}\left[\begin{array}{cc}
4 \bar{\alpha}_{0}+\bar{\lambda}_{0}^{2} & -\bar{\lambda}_{0} \\
-\bar{\lambda}_{0} & 2
\end{array}\right] .
\end{aligned}
$$

Taking the derivative of (17),

$$
\begin{aligned}
\dot{V}= & \frac{l(t)}{2\left|\zeta_{1}\right|} \zeta^{T}\left(A^{T} P+P A\right) \zeta+q_{1} \phi(x, t) \zeta \\
& +\frac{\dot{l}(t)}{2 l(t)} \zeta_{1} q_{2} \zeta,
\end{aligned}
$$

where $q_{1}=\left[-\bar{\lambda}_{0}, 2\right]$ and $q_{2}=\left[4 \bar{\alpha}_{0}+\bar{\lambda}_{0}^{2},-\bar{\lambda}_{0}\right]$.

Since $A$ is a Hurwitz matrix, there exists a positive definite matrix $Q$ such that $A^{T} P+P A=-Q$ and $\lambda_{\min }(P)\|\zeta\|^{2} \leq V \leq$ $\lambda_{\max }(P)\|\zeta\|^{2}$. Equation (18) can be rewritten as

$$
\dot{V} \leq-\frac{l(t)}{2} \frac{\lambda_{\min }(Q)}{\lambda_{\max }^{1 / 2}(P)} V^{1 / 2}+\frac{\sigma_{2}\left\|q_{1}\right\|}{\lambda_{\min }^{1 / 2}(P)} V^{1 / 2}+\Delta V,
$$

where

$$
\begin{aligned}
\Delta V & =\frac{i(t)}{2 l(t)}\left[\left(4 \bar{\alpha}_{0}+\bar{\lambda}_{0}^{2}\right) \zeta_{1}^{2}-2 \bar{\lambda}_{0} \zeta_{1} \zeta_{2}\right] \\
& \leq \frac{i(t)}{2 l(t)}\left[\left(4 \bar{\alpha}_{0}+\bar{\lambda}_{0}^{2}+\frac{\bar{\lambda}_{0}}{2}\right) \zeta_{1}^{2}+\frac{\bar{\lambda}_{0}}{2} \zeta_{2}^{2}\right] \\
& =\frac{i(t)}{2 l(t)} \zeta^{T} \Delta Q \zeta, \\
\Delta Q & =\left[\begin{array}{cc}
4 \bar{\alpha}_{0}+\bar{\lambda}_{0}^{2}+\frac{\bar{\lambda}_{0}}{2} & 0 \\
0 & \frac{\bar{\lambda}_{0}}{2}
\end{array}\right] .
\end{aligned}
$$

With (20), (19) is present as

$$
\begin{aligned}
\dot{V} \leq & -\left(\frac{l(t)}{2} \frac{\lambda_{\min }(Q)}{\lambda_{\max }^{1 / 2}(P)}-\frac{\sigma_{2}\left\|q_{1}\right\|}{\lambda_{\min }^{1 / 2}(P)}\right) V^{1 / 2} \\
& +\frac{i(t)}{2 l(t)} \frac{\lambda_{\max }(\Delta Q)}{\lambda_{\min }(P)} V .
\end{aligned}
$$

For simplicity, we define

$$
\begin{aligned}
& \gamma_{1}=\frac{\lambda_{\text {min }}(Q)}{2 \lambda_{\text {max }}^{1 / 2}(P)}, \\
& \gamma_{2}=\frac{\sigma_{2}\left\|q_{1}\right\|}{\lambda_{\text {min }}^{1 / 2}(P)}, \\
& \gamma_{3}=\frac{\lambda_{\text {max }}(\Delta Q)}{2 \lambda_{\min }(P)} .
\end{aligned}
$$


$\gamma_{1}, \gamma_{2}, \gamma_{3}$ are all positive constants. Thus, (21) is simplified as

$$
\dot{V} \leq-\left(\gamma_{1} l(t)-\gamma_{2}\right) V^{1 / 2}+\gamma_{3} \frac{\dot{l}(t)}{l(t)} V
$$

where $i \geq 0$.

In order to show that the $\dot{V}$ will be negative in finite time, the second time derivative of $V$ is calculated as

$$
\begin{aligned}
\ddot{V} \leq & -\dot{l}(t) V^{1 / 2}-\left(\gamma_{1} l(t)-\gamma_{2}\right) \frac{\dot{V}}{2 V^{1 / 2}}+\gamma_{3} \frac{\dot{l}(t)}{l(t)} \dot{V} \\
& -\gamma_{3} \frac{\dot{l}(t)}{l^{2}(t)} V .
\end{aligned}
$$

If $\dot{V}>0$, the positive term $\gamma_{3}(\dot{l}(t) / l(t)) \dot{V}$ is decreasing and the term $\left(\gamma_{1} l(t)-\gamma_{2}\right)\left(\dot{V} / 2 V^{1 / 2}\right)$ will be positive at some time instant $t=t_{1}$ which dominates the positive term $\gamma_{3}(\dot{l}(t) / l(t)) \dot{V}$, since $l(t)$ is a monotonic nondecreasing function $(\dot{i}(t) \geq 0)$. In this point, we can conclude that after the time $t=t_{1}, \ddot{V}<0$. That is to say $V$ is not increasing faster than a time linear function during time $t \in\left[0, t_{1}\right]$ which can be formulated as

$$
V \leq m t+n
$$

Substituting (25) into (23), we have

$$
\dot{V} \leq-\left(\gamma_{1} l(t)-\gamma_{2}\right) V^{1 / 2}+\gamma_{3} \dot{l}(t) \frac{m t+n}{k t+l(0)},
$$

where $l(0)>0$ is the initial value of the scalar function (14).

The positive term in (26) is bounded,

$$
\gamma_{3} \dot{l}(t) \frac{m t+n}{k t+l(0)} \leq \gamma_{3} \dot{l}(t) \frac{\max \{m, n\}}{\min \{k, l(0)\}} .
$$

Now, we can conclude that after some time $t>t_{1}$ the first term in the right side of (26) will dominate the second term such that

$$
\dot{V} \leq-\theta\left[\gamma_{1} l(t)-\gamma_{2}\right] V^{1 / 2},
$$

with $\theta \in(0,1)$ and $\gamma_{1} l(t)-\gamma_{2}>0$. Therefore, $\sigma$ and $\dot{\sigma}$ converge to zero in finite time. Theorem 4 is proven.

Remark 5. In view of practical implementation, the condition $|\sigma|=0$ in (14) cannot be satisfied due to measurement noise and numerical approximations. The condition $|\sigma|=0$ needs to be modified by dead-zone technique $[41,42]$, such that the dynamic law (14) is practically implementable,

$$
i(t)= \begin{cases}k, & \text { if }|\sigma| \geq \tau \\ 0, & \text { else }\end{cases}
$$

where $\tau$ is a sufficiently small positive value.

\section{Simulation Results and Discussions}

Consider the following nonlinear system [43]:

$$
\begin{aligned}
\dot{x}_{1}= & x_{2}, \\
\dot{x}_{2}= & x_{3}, \\
\dot{x}_{3}= & -b_{1}(t) x_{1}^{2}-b_{2}(t) x_{2}-b_{3}(t) x_{3}+[3+\cos (t)] u \\
& +d(t),
\end{aligned}
$$

where $b_{1}(t)=[1+0.3 \sin (t)], b_{2}(t)=[1.5+0.2 \cos (t)], b_{3}(t)=$ $[1+0.4 \sin (t)]$, and $d(t)=0.05 \sin (0.1 t)$ is considered as an external disturbance.

The desired state trajectory is supposed to be $x_{d}^{T}=$ $[\sin (t), \cos (t),-\sin (t)]$. The sliding-surface function (2) is chosen, and $c^{T}=[10,5,1]$. The initial state vector is $x\left(t_{0}\right)=$ $[0,0,-0.35]^{T}$. The initial value of the sliding variable is taken as $\sigma(0)=-5.35$.

According to (2), the sliding surface is given as

$$
\sigma=c_{1}\left(x_{1}-x_{1 d}\right)+c_{2}\left(x_{2}-x_{2 d}\right)+c_{3}\left(x_{3}-x_{3 d}\right) \text {. }
$$

The time derivative of $\sigma$ is calculated as

$$
\dot{\sigma}=g_{1}(x, t) u+\rho(x, t),
$$

where $g_{1}(x, t)=c_{3}(3+\cos t)$ and $\rho(x, t)=-c^{T} x_{d}+c_{1} x_{2}+$ $c_{2} x_{3}+c_{3} d(t)+c_{3}\left(b_{1}(t) x_{1}^{2}-b_{2}(t) x_{2}-b_{3}(t) x_{3}\right)$.

The adaptive-gain STW control laws (9), (10), (11), and (13) are designed as

$$
\begin{aligned}
& \dot{\sigma}=-\lambda(\sigma, t) g_{1}(x, t)|\sigma|^{1 / 2} \operatorname{sign}(\sigma)+\varphi \\
& \dot{\varphi}=-\alpha(\sigma, t) g_{1}(x, t) \operatorname{sign}(\sigma)+\phi(x, t),
\end{aligned}
$$

where the adaptive-gain $\lambda(\sigma, t)$ and $\alpha(\sigma, t)$ dynamics follow (29) and the values of the parameters of the adaptive-gain law have been taken as $\tau=0.005, \lambda_{0}=2.5, \alpha_{0}=8$, and $k=5$. These parameters are tuned to get sufficiently accurate and fast convergence. Finally, the adaptive-gain dynamic law (29) becomes

$$
i(t)= \begin{cases}5, & \text { if }|\sigma| \geq 0.005 \\ 0, & \text { else. }\end{cases}
$$

Figures 1-3 show the state performance of system (3) in the presence of disturbance $d(t)$. The time response of the sliding surface function $\sigma$ is shown in Figure 4. It can be easily found that $\sigma$ converges to zero in finite time. From Figure 5, we can see that the control input is smooth which is suitable for real applications. Figure 6 shows that the adaptive law of (34) is effective in the presence of external disturbance $d(t)$.

Remark 6. In order to avoid high frequency control activity, the boundary layer technique is employed during the simulation. Thus, the saturation function sat $(\sigma / \Phi)$ is used to replace the function $\operatorname{sign}(\sigma / \Phi)$, where $\Phi$ is the boundary layer width; that is,

$$
\operatorname{sat}\left(\frac{\sigma}{\Phi}\right)= \begin{cases}\frac{\sigma}{\Phi}, & \text { if }\left|\frac{\sigma}{\Phi}\right| \geq 1 \\ \operatorname{sign}\left(\frac{\sigma}{\Phi}\right), & \text { else. }\end{cases}
$$



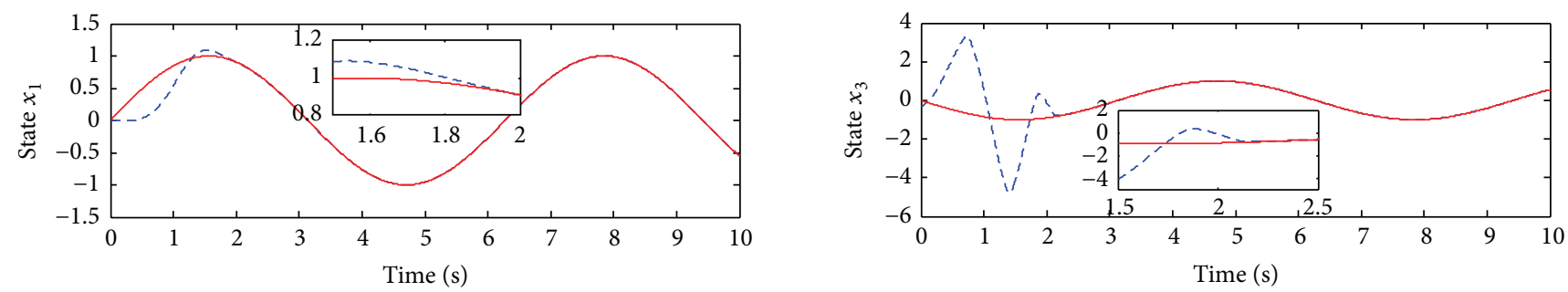

$$
\begin{aligned}
& -x_{1} \\
& -x_{1 d}
\end{aligned}
$$

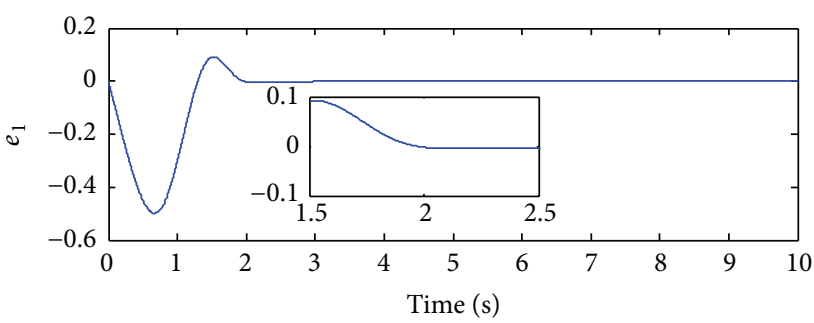

$$
-x_{1}-x_{1 d}
$$

FIGURE 1: The performance of state $x_{1}$ and its error $e_{1}$.
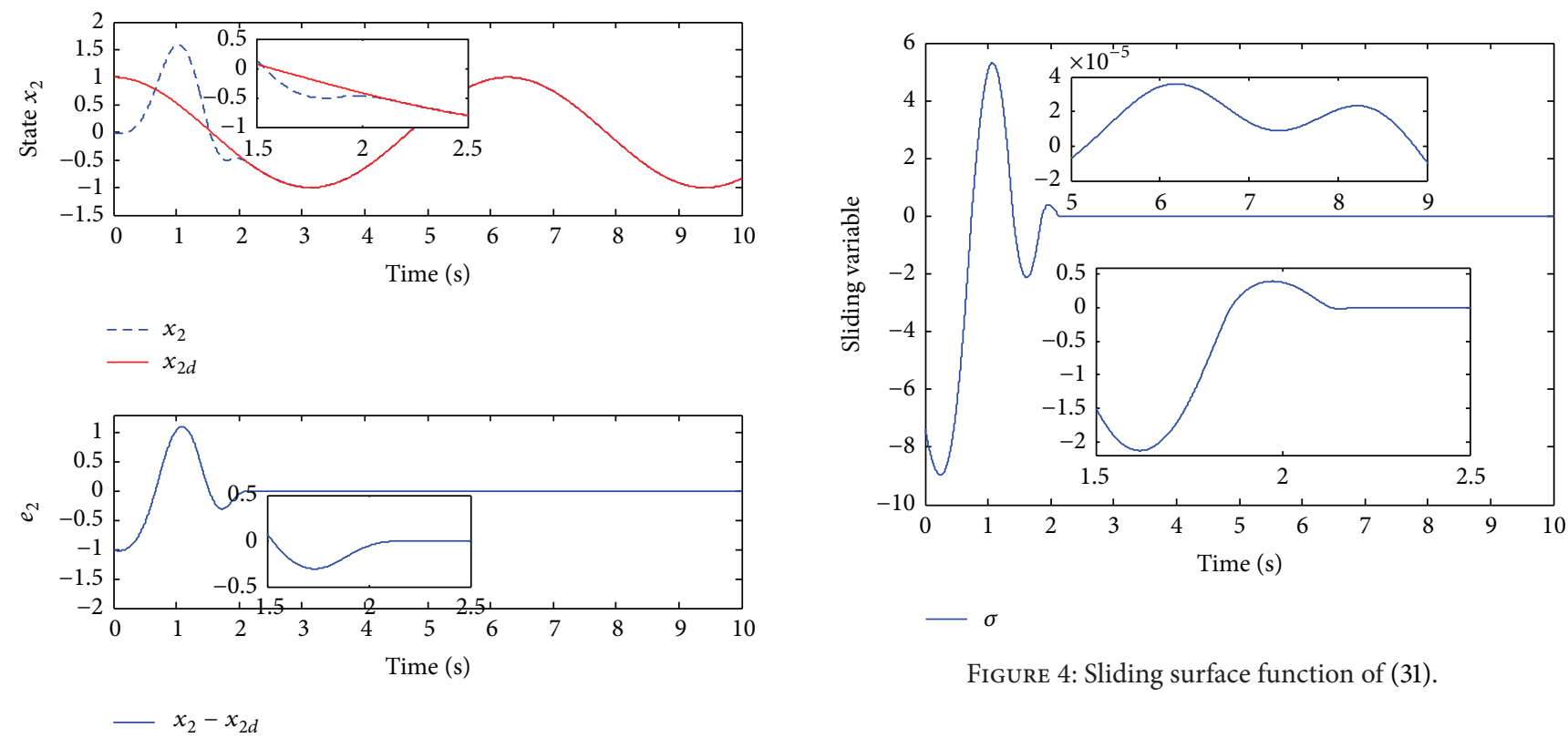

FIGURE 4: Sliding surface function of (31).

FIGURE 2: The performance of state $x_{2}$ and its error $e_{2}$.

\section{Conclusions}

Adaptive second-order sliding mode control design is studied for a class of nonlinear systems with unknown inputs. In real applications, the upper boundary of uncertainty is difficult to obtain, which is required for calculating control gains of super-twisting sliding mode control. To solve this problem, a simple control design method is proposed based on Lyapunov function. The idea is very simple; the gains are increased according to a dynamic law until the sliding mode is attained

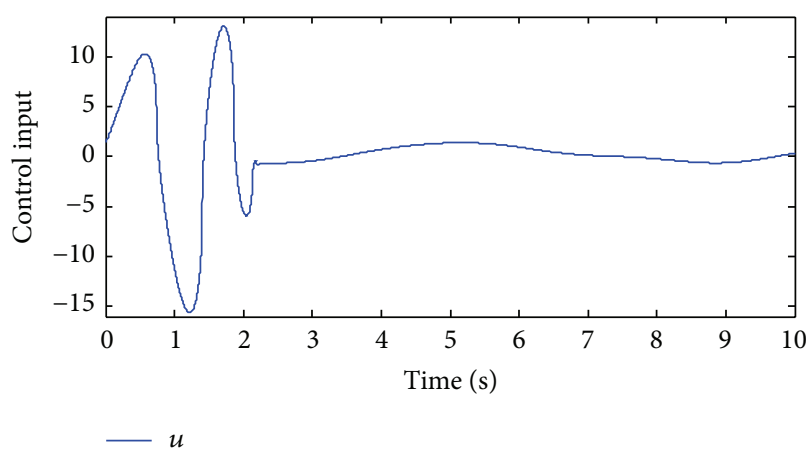

FIGURE 5: Control input $u$ versus time(s). 


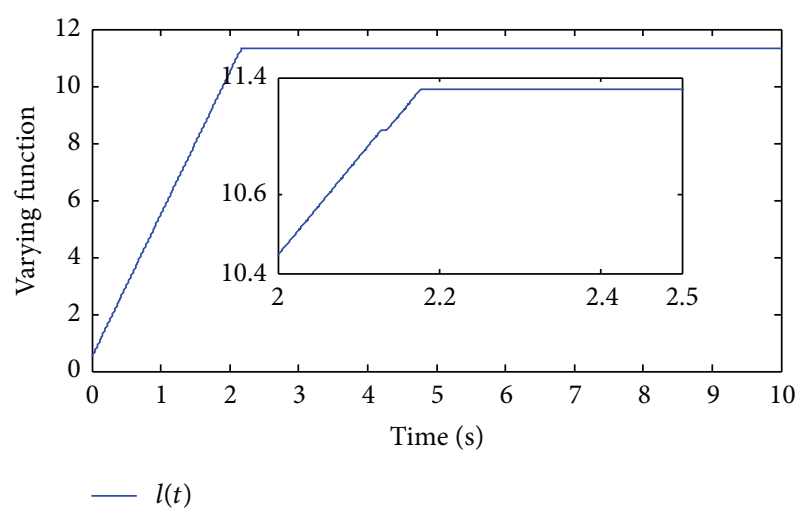

Figure 6: Time varying function $l(t)$.

and stops increasing thereafter. The proposed approach has two advantages:

(i) Only one parameter $k$ has to be tuned.

(ii) A priori knowledge of the uncertainty bound is not required.

\section{Conflict of Interests}

The authors declare that there is no conflict of interests regarding the publication of this paper.

\section{Acknowledgments}

This work was supported by Zhejiang Provincial Natural Science Foundation of China (no. LQ14F010001), Ningbo City Natural Science Foundation of China (no. 2013A610114, no. 2014A610088, and no. 2013A610150), Key Scientific and Technological Projects of Ningbo (no. 2014B92001), and the Fundamental Research Funds for the Central Universities (Grant no. HIT. NSRIF201625).

\section{References}

[1] A. Levant, "Sliding order and sliding accuracy in sliding mode control," International Journal of Control, vol. 58, no. 6, pp. 12471263, 1993.

[2] A. Šabanovic, "Variable structure systems with sliding modes in motion control-a survey," IEEE Transactions on Industrial Informatics, vol. 7, no. 2, pp. 212-223, 2011.

[3] X.-G. Yan, C. Edwards, and S. K. Spurgeon, "Output feedback sliding mode control for non-minimum phase systems with non-linear disturbances," International Journal of Control, vol. 77, no. 15, pp. 1353-1361, 2004.

[4] X.-G. Yan, S. K. Spurgeon, and C. Edwards, "Decentralised stabilisation for nonlinear time delay interconnected systems using static output feedback," Automatica, vol. 49, no. 2, pp. 633641, 2013.

[5] X.-G. Yan, S. K. Spurgeon, and C. Edwards, "State and parameter estimation for nonlinear delay systems using sliding mode techniques," IEEE Transactions on Automatic Control, vol. 58, no. 4, pp. 1023-1029, 2013.
[6] R. J. Talj, D. Hissel, R. Ortega, M. Becherif, and M. Hilairet, "Experimental validation of a PEM fuel-cell reduced-order model and a moto-compressor higher order sliding-mode control," IEEE Transactions on Industrial Electronics, vol. 57, no. 6, pp. 1906-1913, 2010.

[7] S. Laghrouche, J. Liu, F. Ahmed, M. Harmouche, and M. Wack, "Adaptive second-order sliding mode observer-based fault reconstruction for pem fuel cell air-feed system," IEEE Transactions on Control Systems Technology, vol. 23, no. 3, pp. 1098-1109, 2014.

[8] W. Garcia-Gabin, F. Dorado, and C. Bordons, "Real-time implementation of a sliding mode controller for air supply on a PEM fuel cell," Journal of Process Control, vol. 20, no. 3, pp. 325-336, 2010.

[9] J. Liu, S. Laghrouche, F. S. Ahmed, and M. Wack, "PEM fuel cell air-feed system observer design for automotive applications: an adaptive numerical differentiation approach," International Journal of Hydrogen Energy, vol. 39, no. 30, pp. 17210-17221, 2014.

[10] C. Kunusch, P. F. Puleston, M. A. Mayosky, and L. Fridman, "Experimental results applying second order sliding mode control to a PEM fuel cell based system," Control Engineering Practice, vol. 21, no. 5, pp. 719-726, 2013.

[11] C. Kunusch, P. F. Puleston, M. A. Mayosky, and J. Riera, "Sliding mode strategy for PEM fuel cells stacks breathing control using a super-twisting algorithm," IEEE Transactions on Control Systems Technology, vol. 17, no. 1, pp. 167-174, 2009.

[12] J. Liu, S. Laghrouche, and M. Wack, “Observer-based higher order sliding mode control of power factor in three-phase AC/DC converter for hybrid electric vehicle applications," International Journal of Control, vol. 87, no. 6, pp. 1117-1130, 2014.

[13] Y. B. Shtessel, A. S. Zinober, and I. A. Shkolnikov, "Sliding mode control of boost and buck-boost power converters using the dynamic sliding manifold," International Journal of Robust and Nonlinear Control, vol. 13, no. 14, pp. 1285-1298, 2003.

[14] A. Abrishamifar, A. A. Ahmad, and M. Mohamadian, "Fixed switching frequency sliding mode control for single-phase unipolar inverters," IEEE Transactions on Power Electronics, vol. 27, no. 5, pp. 2507-2514, 2012.

[15] J. Liu, S. Laghrouche, M. Harmouche, and M. Wack, "Adaptivegain second-order sliding mode observer design for switching power converters," Control Engineering Practice, vol. 30, pp. 124131, 2014.

[16] E. Vidal-Idiarte, L. Martinez-Salamero, J. Calvente, and A. Romero, "An $H_{\infty}$ control strategy for switching converters in sliding-mode current control," IEEE Transactions on Power Electronics, vol. 21, no. 2, pp. 553-556, 2006.

[17] B. Xiao, Q.-L. Hu, and G. Ma, "Adaptive sliding mode backstepping control for attitude tracking of flexible spacecraft under input saturation and singularity," Proceedings of the Institution of Mechanical Engineers, Part G: Journal of Aerospace Engineering, vol. 224, no. 2, pp. 199-214, 2010.

[18] B. Xiao, Q. Hu, and Y. Zhang, "Adaptive sliding mode fault tolerant attitude tracking control for flexible spacecraft under actuator saturation," IEEE Transactions on Control Systems Technology, vol. 20, no. 6, pp. 1605-1612, 2012.

[19] V. Parra-Vega and G. Hirzinger, "Chattering-free sliding mode control for a class of nonlinear mechanical systems," International Journal of Robust and Nonlinear Control, vol. 11, no. 12, pp. 1161-1178, 2001. 
[20] A. Ferreira, F. J. Bejarano, and L. M. Fridman, "Robust control with exact uncertainties compensation: with or without chattering?" IEEE Transactions on Control Systems Technology, vol. 19, no. 5, pp. 969-975, 2011.

[21] H. Lee and V. I. Utkin, "Chattering suppression methods in sliding mode control systems," Annual Reviews in Control, vol. 31, no. 2, pp. 179-188, 2007.

[22] I. Boiko and L. Fridman, "Analysis of chattering in continuous sliding-mode controllers," IEEE Transactions on Automatic Control, vol. 50, no. 9, pp. 1442-1446, 2005.

[23] Y. Zhao, C. Cachard, and H. Liebgott, "Automatic needle detection and tracking in 3D ultrasound using an ROI-based RANSAC and Kalman method," Ultrasonic Imaging, vol. 35, no. 4, pp. 283-306, 2013.

[24] S. Yin, H. Luo, and S. X. Ding, "Real-time implementation of fault-tolerant control systems with performance optimization," IEEE Transactions on Industrial Electronics, vol. 61, no. 5, pp. 2402-2411, 2014.

[25] C. Cachard, Y. Zhao, and H. Liebgott, "Comparison of the existing tool localisation methods on two-dimensional ultrasound images and their tracking results," IET Control Theory \& Applications, vol. 9, no. 7, pp. 1124-1134, 2015.

[26] S. Yin, X. Zhu, and O. Kaynak, "Improved pls focused on keyperformance-indicator-related fault diagnosis," IEEE Transactions on Industrial Electronics, vol. 62, no. 3, pp. 1651-1658, 2015.

[27] H. Elmali and N. Olgac, "Sliding mode control with perturbation estimation (SMCPE): a new approach," International Journal of Control, vol. 56, no. 4, pp. 923-941, 1992.

[28] J. A. Burton and A. S. I. Zinober, "Continuous approximation of variable structure control," International Journal of Systems Science, vol. 17, no. 6, pp. 875-885, 1986.

[29] A. Levant, "Higher-order sliding modes, differentiation and output-feedback control," International Journal of Control, vol. 76, no. 9-10, pp. 924-941, 2003.

[30] J. Liu, Y. Zhao, B. Geng, and B. Xiao, "Adaptive second order sliding mode control of a fuel cell hybrid system for electric vehicle applications," Mathematical Problems in Engineering. In press.

[31] V. I. Utkin and A. S. Poznyak, "Adaptive sliding mode control with application to super-twist algorithm: equivalent control method," Automatica, vol. 49, no. 1, pp. 39-47, 2013.

[32] Y. Shtessel, M. Taleb, and F. Plestan, "A novel adaptive-gain supertwisting sliding mode controller: methodology and application," Automatica, vol. 48, no. 5, pp. 759-769, 2012.

[33] J.-J. Slotine and S. S. Sastry, "Tracking control of nonlinear systems using sliding surfaces, with application to robot manipulators," International Journal of Control, vol. 38, no. 2, pp. 465492, 1983

[34] W.-D. Chang, R.-C. Hwang, and J.-G. Hsieh, "Application of an auto-tuning neuron to sliding mode control," IEEE Transactions on Systems, Man and Cybernetics Part C: Applications and Reviews, vol. 32, no. 4, pp. 517-522, 2002.

[35] T.-S. Chiang, C.-S. Chiu, and P. Liu, "Adaptive TS-FNN control for a class of uncertain multi-time-delay systems: the exponentially stable sliding mode-based approach," International Journal of Adaptive Control and Signal Processing, vol. 23, no. 4, pp. 378-399, 2009.

[36] F. Da and W. Song, "Fuzzy neural networks for direct adaptive control," IEEE Transactions on Industrial Electronics, vol. 50, no. 3, pp. 507-513, 2003.
[37] V. Nekoukar and A. Erfanian, "Adaptive fuzzy terminal sliding mode control for a class of MIMO uncertain nonlinear systems," Fuzzy Sets and Systems, vol. 179, no. 1, pp. 34-49, 2011.

[38] H. Alli and O. Yakut, "Fuzzy sliding-mode control of structures," Engineering Structures, vol. 27, no. 2, pp. 277-284, 2005

[39] J. Davila, L. Fridman, and A. Levant, "Second-order slidingmode observer for mechanical systems," IEEE Transactions on Automatic Control, vol. 50, no. 11, pp. 1785-1789, 2005.

[40] A. F. Filippov, "Differential equations with discontinuous righthand side," Matematicheskii Sbornik, vol. 93, no. 1, pp. 99-128, 1960.

[41] J.-J. E. Slotine and W. Li, Applied Nonlinear Control, vol. 1, Prentice Hall, Englewood Cliffs, NJ, USA, 1991.

[42] A. Pisano and E. Usai, "Globally convergent real-time differentiation via second order sliding modes," International Journal of Systems Science, vol. 38, no. 10, pp. 833-844, 2007.

[43] Y.-J. Huang, T.-C. Kuo, and S.-H. Chang, "Adaptive slidingmode control for nonlinear systems with uncertain parameters," IEEE Transactions on Systems, Man, and Cybernetics, Part B: Cybernetics, vol. 38, no. 2, pp. 534-539, 2008. 


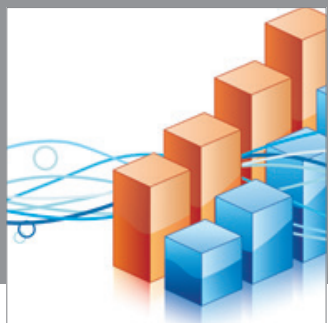

Advances in

Operations Research

mansans

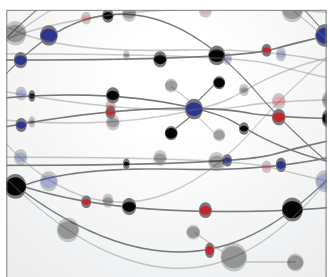

The Scientific World Journal
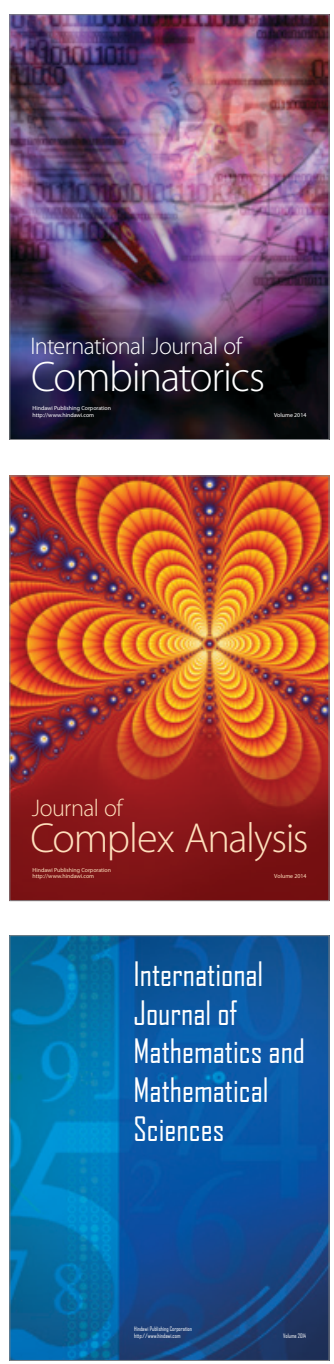
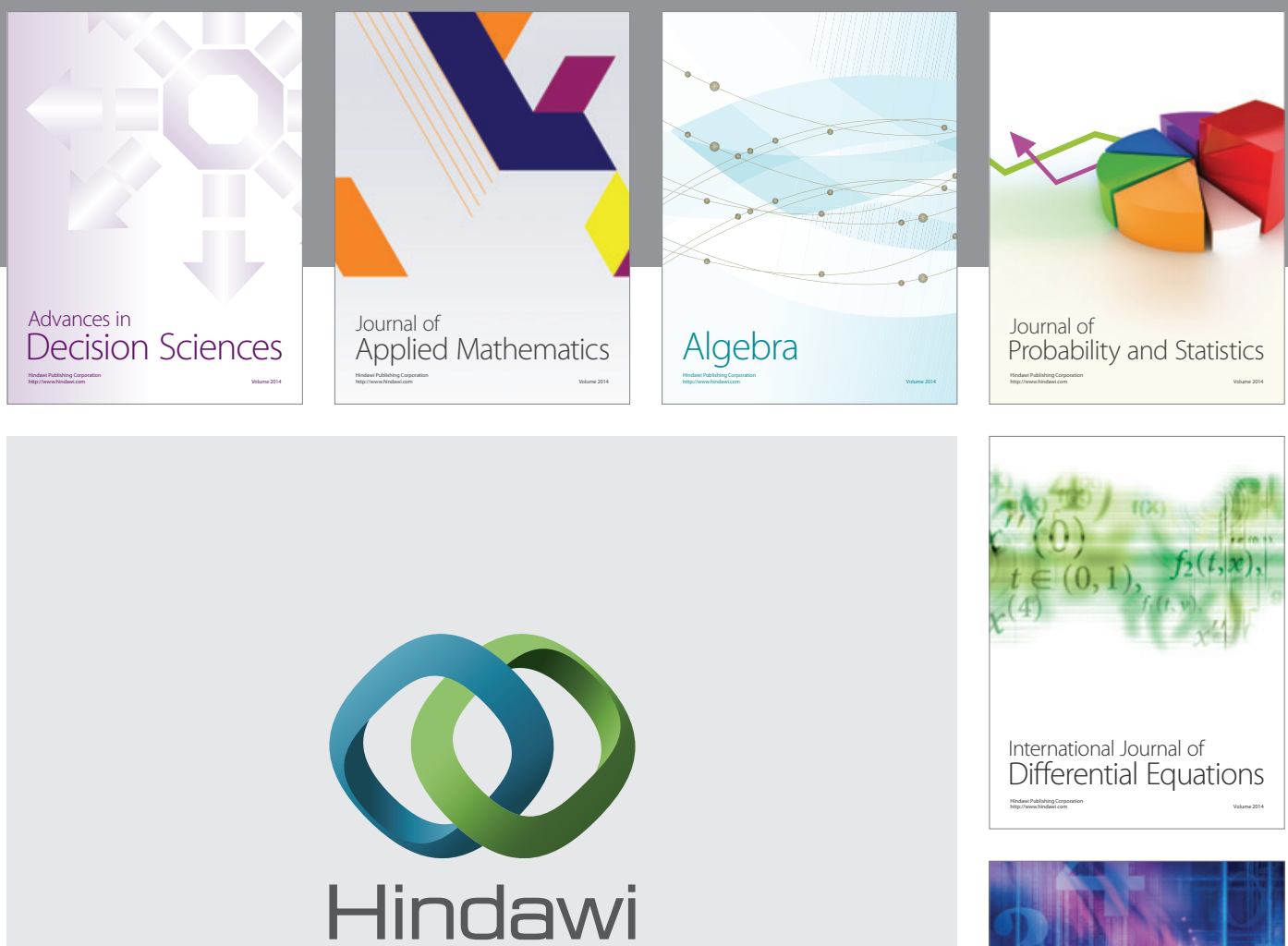

Submit your manuscripts at http://www.hindawi.com
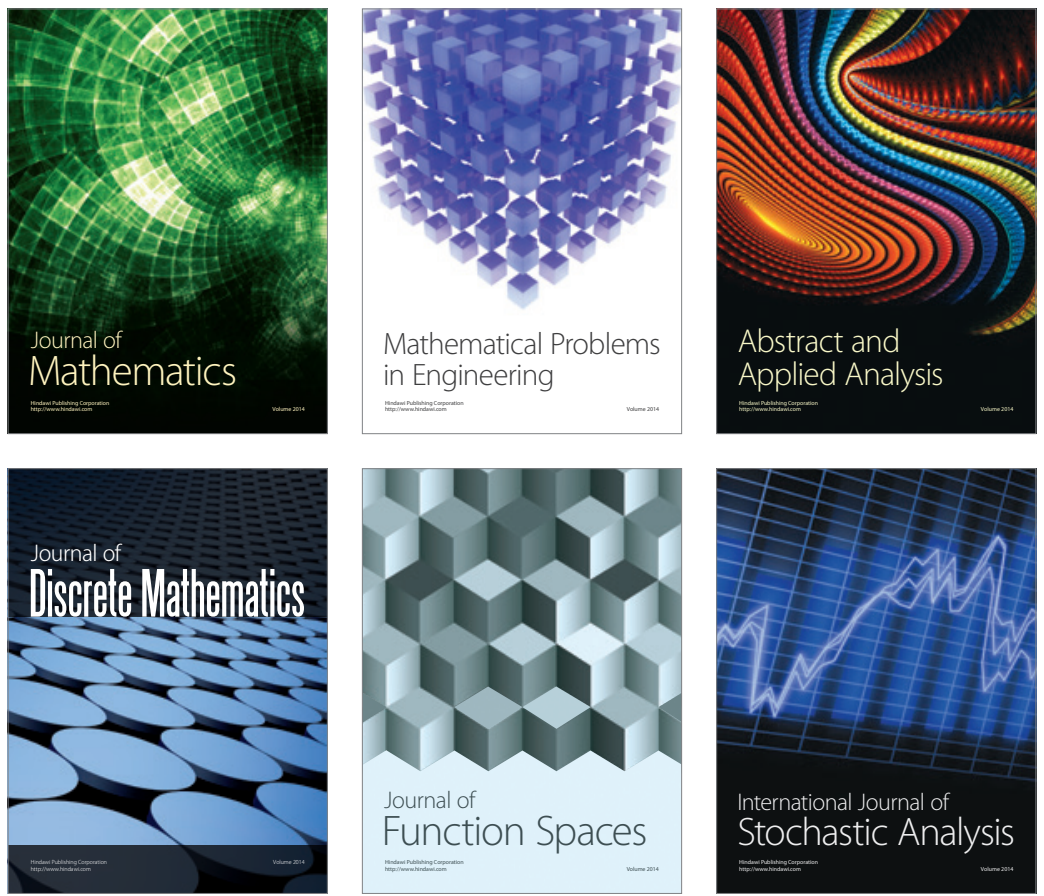

Journal of

Function Spaces

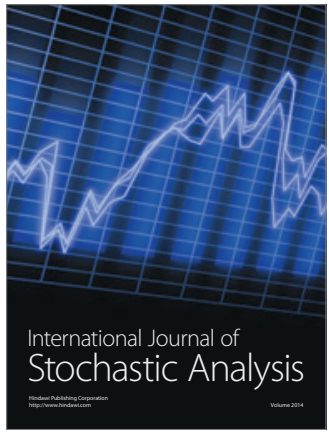

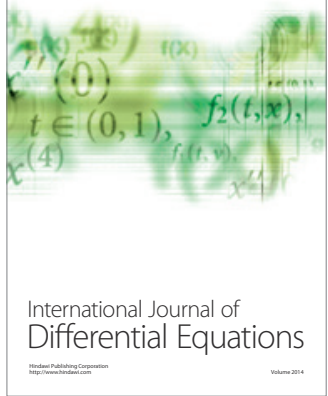
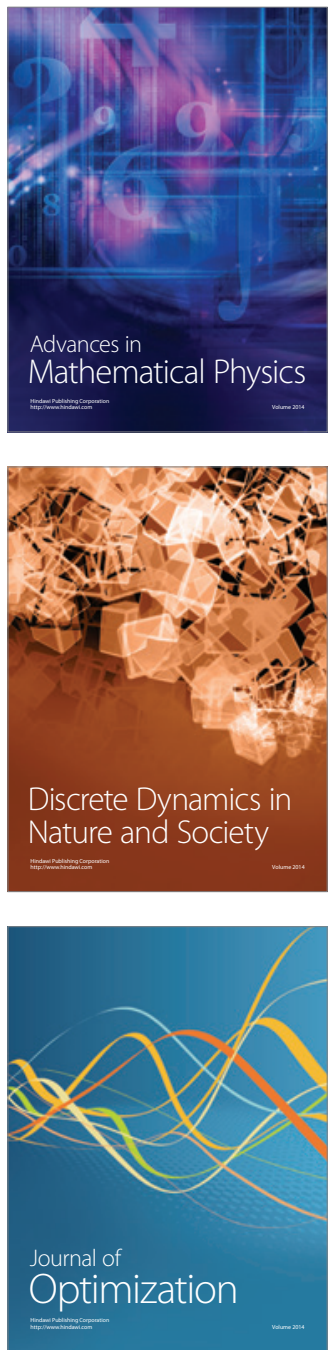